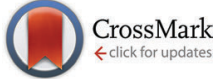

Cite this: J. Mater. Chem. C, 2016 4, 9179

Received 3rd August 2016

Accepted 8th September 2016

DOI: $10.1039 / \mathrm{c} 6 \mathrm{tc} 03342 \mathrm{c}$

www.rsc.org/MaterialsC

\section{Evolution of $\mathrm{CsPbBr} 3$ nanocrystals upon post- synthesis annealing under an inert atmosphere $\dagger$}

\author{
F. Palazon, F. Di Stasio, S. Lauciello, R. Krahne, M. Prato* and L. Manna*
}

\begin{abstract}
Annealing a film of $\mathrm{CsPBBr}_{3}$ nanocrystals (NCs) leads to the removal of surface ligands and ripening of the NCs below $200^{\circ} \mathrm{C}$. This results in a reduced photoluminescence quantum yield due to the formation of trap states while on the other hand the conductive properties of the films are stabilized, with a significantly stronger photocurrent after annealing.
\end{abstract}

Colloidal perovskite quantum dots (QDs), and in particular colloidal $\mathrm{CsPBBr}_{3}$ nanocrystals (NCs), have been gathering considerable attention in the last couple of years, ${ }^{1-4}$ as papers in rapid succession have reported electroluminescence, ${ }^{5}$ lasing, ${ }^{6}$ fast anion-exchange reactions, ${ }^{7,8}$ fluorescence blinking and photoactivation, ${ }^{9}$ energy transfer, ${ }^{10}$ single-photon emission, ${ }^{11}$ shape control leading to bandgap tuning through quantum confinement, ${ }^{12,13}$ and stability in water. ${ }^{14,15}$ The relatively low cost of fabrication of these nanomaterials has already enabled the development of gram-scale synthesis methods ${ }^{16}$ and their implementation in different optoelectronic devices, such as LEDs. ${ }^{16-18}$ To date, the pace at which new potential applications based on $\mathrm{CsPbBr}_{3}$ NCs are being proposed is not matched by studies on how these NCs, which are much more labile than conventional semiconductor QDs, evolve under different external agents such as heat, humidity or UV exposure. Here temperature is probably the most straightforward and widespread factor to take into account. To date, the influence of temperature on the shape and optical properties of cesium lead halide NCs has been studied mainly as a parameter that is tuned during the synthesis of the $\mathrm{NCs},{ }^{19}$ while many more studies have been carried out on bulk perovskite films. The thermal stability of the films was found to depend on the chemical composition as well as under annealing conditions (in air, nitrogen or vacuum). ${ }^{20-22}$ Conings et al..$^{23}$ demonstrated for example that annealing at $85{ }^{\circ} \mathrm{C}$ caused significant degradation of hybrid organic-inorganic perovskite thin films even

Nanochemistry Department, Istituto Italiano di Tecnologia, Via Morego 30, 16163 Genova, Italy.E-mail: mirko.prato@iit.it, liberato.manna@iit.it $\dagger$ Electronic supplementary information (ESI) available: Methods, PL decay times and HRSEM characterization. See DOI: 10.1039/c6tc03342c under an inert atmosphere. The instability of methylammonium lead trihalide films upon annealing ${ }^{21}$ is one of the reasons that encouraged the research on the more stable, fully inorganic cesium lead trihalide $\left(\mathrm{CsPbX}_{3}\right)$ counterparts. ${ }^{20,24}$ According to Sutton et al. ${ }^{20} \mathrm{CsPbX}_{3}$ thin films are characterized by both optimal crystallinity and optical performances when annealed at $350{ }^{\circ} \mathrm{C}$. Colloidal $\mathrm{CsPbX}_{3}$ NCs are fundamentally different from their thin film counterparts, due to their much higher surface to volume ratio and their high organic content arising from the molecular ligands that coat their surface. Therefore, it is not possible to extrapolate the behaviour upon annealing of a "film" of deposited NCs on a substrate from the reported results for bulk films. The lack of extensive studies in this direction is notable, as the development of perovskite NC-based optoelectronic devices often involves heating during fabrication (e.g., lithography resist annealing $)^{25}$ or during operation (i.e., Joule effect). In this work, we fill this gap by studying the effect of annealing at different temperatures (from $50{ }^{\circ} \mathrm{C}$ to $200{ }^{\circ} \mathrm{C}$ ) under an inert atmosphere.

In the typical colloidal synthesis as described by Protesescu et $\mathrm{al}^{2}{ }^{2}$ $\mathrm{CsPbBr}_{3} \mathrm{NCs}$ are stabilized with long chain organic ligands (oleylamine - OlAm - and oleic acid - OlAc - dissolved in octadecene - ODE -, each molecule having an 18 carbon chain). This is not anomalous in the colloidal synthesis of inorganic NCs, but what is peculiar to perovskite NCs is their instability in polar solvents, which makes cleaning of the sample rather difficult. ${ }^{5,6,26}$ Therefore, NCs are typically centrifuged without antisolvent and redispersed in toluene after discarding the supernatant. ${ }^{5,18}$ This procedure leads to a poor cleaning efficiency, leaving residues of unbound ligands as well as ODE in the final solution, as also noted by others. ${ }^{5,6,26} \mathrm{~A}$ large excess of these residues can result in a drop-cast film that remains partly fluid (gel-like) and not fully dried under ambient conditions. We show herein that annealing causes the removal of organic residues and part of the ligands at the surface of the NCs, which in turn affects the optoelectronic properties of the film. From $50{ }^{\circ} \mathrm{C}$ to $200{ }^{\circ} \mathrm{C}$, a drop of the photoluminescence quantum yield (PLQY) is observed, accompanied by faster photoluminescence (PL) decay owing to the creation of trap states at the surface of 

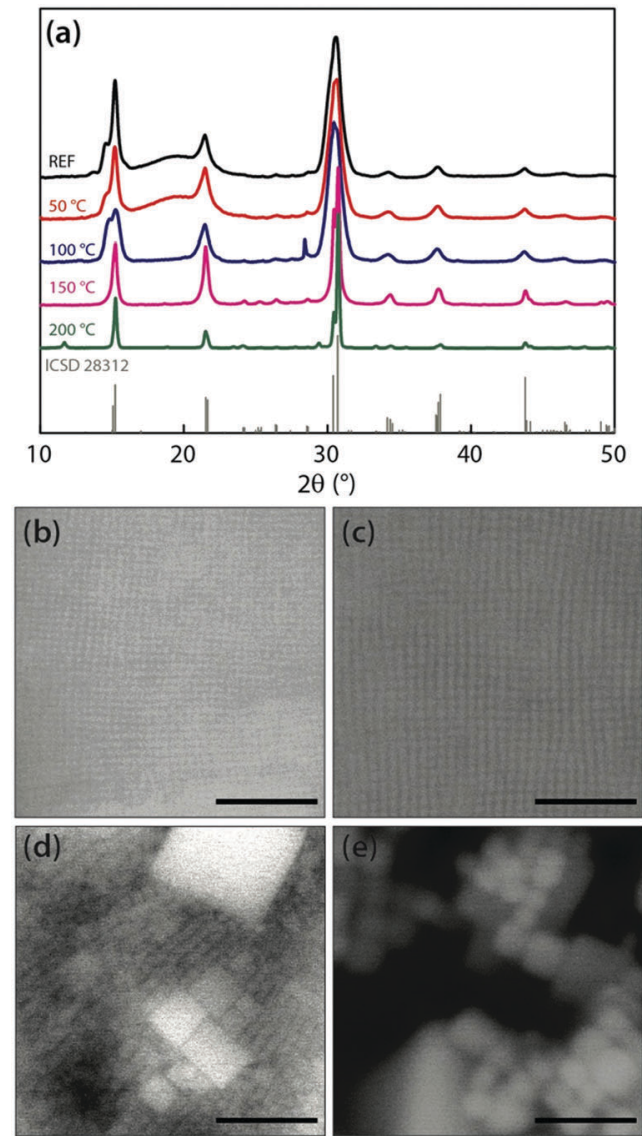

Fig. 1 (a) XRD patterns and (b-e) HRSEM images of samples annealed at $50{ }^{\circ} \mathrm{C}$ (b), $100{ }^{\circ} \mathrm{C}$ (c), $150{ }^{\circ} \mathrm{C}$ (d), and $200{ }^{\circ} \mathrm{C}$ (e); scale bar is $100 \mathrm{~nm}$. Characterization at different temperatures was realized on different dropcast films prepared under the same conditions (except for temperature) and from the same colloidal solution (see experimental section for more details).

the NCs. The $\mathrm{CsPbBr}_{3}$ NC films also demonstrated a stable electrical conductivity and increased photocurrent following the annealing steps. At high temperatures (150-200 $\left.{ }^{\circ} \mathrm{C}\right)$ ripening of the NCs into larger crystals is observed.

When annealing of the film is performed under ambient pressure in nitrogen, a significant portion of the organic component can be removed from the film at $100{ }^{\circ} \mathrm{C}$. This can be seen by the disappearance of the broad feature around $20^{\circ}$ in the XRD pattern (Fig. 1a), which is assigned to oleylamine and oleic $\operatorname{acid}^{27}$ and is further confirmed by XPS analysis (Fig. S2, ESI $\dagger)$. The morphology of the film remains mostly unchanged up to $100-150{ }^{\circ} \mathrm{C}$ (Fig. $1 \mathrm{~b}-\mathrm{d}$ ) and consistent with that of a dried film of $8 \mathrm{~nm}$ cubes prepared at room temperature (Fig. S1, ESI $\dagger$ ). This may suggest that the organic fraction that is removed from the film is primarily coming from free ligands and solvent residues. When annealing is performed at higher temperatures (150-200 $\left.{ }^{\circ} \mathrm{C}\right)$, further changes become apparent. At the maximum temperature applied $\left(200{ }^{\circ} \mathrm{C}\right)$ a small peak appears at $11.67^{\circ}$, which may be linked to the presence of $\mathrm{CsPb}_{2} \mathrm{Br}_{5}$ species. $^{28}$ The low intensity of the peak, however, indicates that these species are residual, and the positions and
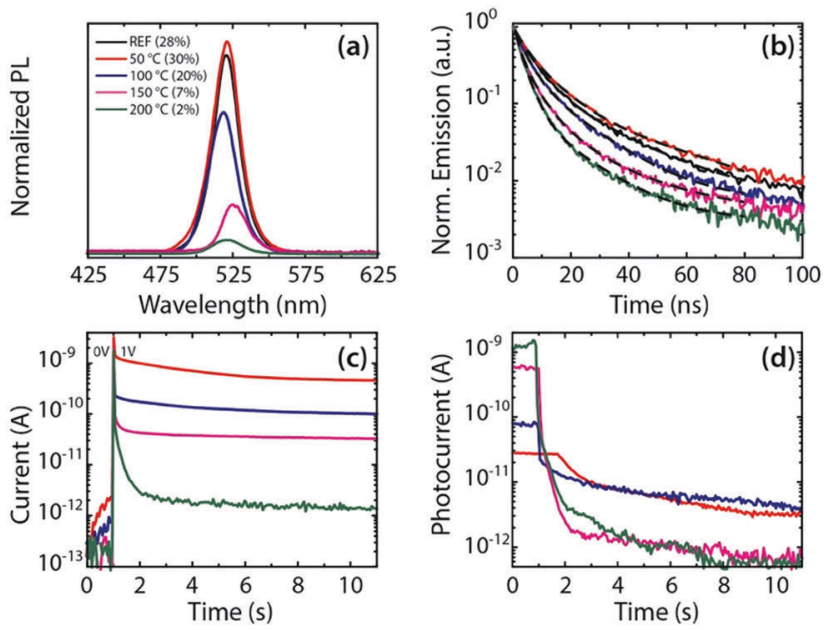

Fig. 2 (a) Normalized PL spectra for films annealed at different temperatures, the PLQY is given in the brackets in the legend. (b) Time traces showing the PL decay recorded at the center wavelength of the PL peak. (c, d) Current vs. time, where bias voltage (c) or light source (d) were abruptly turned off after $1 \mathrm{~s}$. Color coding given in (a) applies to all panels $(a-d)$. The different films were prepared from the same colloidal solution under identical conditions.

relative intensities of the main peaks in the XRD patterns (Fig. 1a) remain the same, corresponding to the orthorhombic $\mathrm{CsPbBr}_{3}$ perovskite (reference pattern: ICSD 28312). Nonetheless, these are much narrower, meaning that the crystals have started ripening. Indeed, HRSEM (Fig. 1b-e) reveals much bigger crystals, with basically no trace of the original $8 \mathrm{~nm}$ cubes at $200{ }^{\circ} \mathrm{C}$. We can imply that this further ripening is aided by the additional removal of surface ligands that promotes coalescence of the NCs.

The optoelectronic properties of the films (Fig. 2) undergo significant changes already after annealing at comparatively low temperatures, i.e. below $150{ }^{\circ} \mathrm{C}$. The PL spectra and PL decay after annealing at different temperatures are shown in Fig. 2a and b. The PL peak appears at around $521 \mathrm{~nm}$ with slight variations in the wavelength $(<5 \mathrm{~nm})$. The peak is especially red-shifted at $150{ }^{\circ} \mathrm{C}(525 \mathrm{~nm})$, which may indicate a loss of quantum confinement as the NCs start coalescing at this temperature. However, the further blue-shift to the original value at $200{ }^{\circ} \mathrm{C}$ seems to contradict this. It is possible, however, that different transformations start taking place at this temperature (see discussion in Fig. 1a above), which presently are not completely elucidated. The significant changes appear though in PLQY and PL decay times. Both PLQY (Fig. 2a) and PL decay lifetimes (Fig. 2b and Table S1, ESI $\dagger$ ) show a slight increase ( $28 \%$ to $30 \%, 8.6 \mathrm{~ns}$ to $13.9 \mathrm{~ns}$, respectively) for annealing at $50{ }^{\circ} \mathrm{C}$. The increase in PL decay time under mild annealing indicates a partial suppression of fast non-radiative channels that is beneficial for the PLQY. Annealing at $100{ }^{\circ} \mathrm{C}$ or above results in a more efficient removal of organic components (as shown in Fig. 1) and PLQY and PL lifetime drop below the values of the untreated films. PLQY drops roughly linearly from $30 \%$ (at $50{ }^{\circ} \mathrm{C}$ ) to around $2 \%$ (at $200{ }^{\circ} \mathrm{C}$ ), which is accompanied by a decrease in the PL lifetime from 8.57 to 4 ns at $200{ }^{\circ} \mathrm{C}$ (weighted average lifetime $\langle\tau\rangle$, see Table S1, ESI $\dagger$ ). Apart from 
synthetic residues, such organic components can also include the ligands that passivate the NC surface. The decrease in PLQY and PL lifetimes can be explained by the formation of trap states induced by ligand removal. ${ }^{29}$

One would expect a reduction in the organic content in a NC film to enhance its charge transport, as reported for other inorganic colloidal NCs. ${ }^{30}$ We measured the electrical current response of the NC films deposited on interdigitated Au electrode arrays before and after the annealing processes described before. Untreated films did not allow recording stable or reproducible data, and current-voltage curves measured on films annealed at low temperature showed large hysteresis and often instabilities. We therefore investigated the effect of annealing on the stability and time response of the current and photocurrent of the films. In Fig. $2 c$ the current is recorded over time while an electrical bias voltage is abruptly switched from 0 to $1 \mathrm{~V}$ at $t=1 \mathrm{~s}$. All current traces show a strong and fast overshoot within ms that can be attributed to the electrical measurement circuit. After that, we observe a decrease in decay time with the increasing annealing temperature that is accompanied by a reduction of the steady state current. We conclude that the annealing removes organic components from the film that contribute to conduction with a very slow time response, and that the removal is more efficient for higher annealing temperatures. The response of the photocurrent $\left(I_{\text {light }}-I_{\text {dark }}\right)$ is shown in Fig. 2 d. Here the current is measured under white light illumination that is abruptly switched off at $t=1 \mathrm{~s}$. The $50{ }^{\circ} \mathrm{C}$ film exhibits only low photocurrent that decays slowly (over several seconds) once the light is switched off. Higher annealing temperatures lead to orders of magnitude higher photocurrents and significantly faster photocurrent decays. Here the ligand removal caused by higher annealing temperatures can create the additional trap states which can provide more efficient pathways for the extraction of photogenerated charges. The photocurrent decay times in the range of hundreds of milliseconds to some seconds, as observed in Fig. 2d for higher annealing temperatures, are in line with those observed from NC films of other materials. ${ }^{31}$

\section{Conclusions}

In conclusion, the evolution of the chemical and optoelectronic properties on a drop-cast film of $\mathrm{CsPbBr}_{3} \mathrm{NCs}_{\text {upon annealing }}$ has been described. Below $100{ }^{\circ} \mathrm{C}$, the morphology of the initial $8 \mathrm{~nm}$ cubes was preserved and only the organic fraction was affected. Here, bound and unbound ligands still present in the pristine film from colloidal synthesis were removed upon annealing. Annealing above $150{ }^{\circ} \mathrm{C}$ led to the coalescence of NCs, with preservation of their orthorhombic $\mathrm{CsPbBr}_{3}$ phase. These structural and chemical transformations resulted in drastic changes in the optoelectronic properties of the drop-cast film. At $50{ }^{\circ} \mathrm{C}$, PLQY increased from $28 \%$ to $30 \%$ demonstrating a positive effect from the treatment. However, at higher temperatures PLQY and PL decay times dropped significantly $\left(2 \%\right.$ at $\left.200{ }^{\circ} \mathrm{C}\right)$. This is related to the creation of trap states at the surface of the NCs, originated by the stripping of the ligands. Untreated films manifested unstable and irreproducible conductivity, and annealing resulted in a more stable current with lower magnitude. The photocurrent of the films was increased by more than one order of magnitude using the annealing treatment. Our findings show that annealing under an inert atmosphere improves the performance of $\mathrm{CsPBr}_{3} \mathrm{NCs}$ for optoelectronic applications.

\section{Acknowledgements}

The research leading to these results has received funding from the 7th European Community Framework Programme under grant agreement no. 614897 (ERC Consolidator Grant “TRANS-NANO”).

\section{Notes and references}

1 L. C. Schmidt, A. Pertegas, S. Gonzalez-Carrero, O. Malinkiewicz, S. Agouram, G. Minguez Espallargas, H. J. Bolink, R. E. Galian and J. Perez-Prieto, J. Am. Chem. Soc., 2014, 136, 850-853.

2 L. Protesescu, S. Yakunin, M. I. Bodnarchuk, F. Krieg, R. Caputo, C. H. Hendon, R. X. Yang, A. Walsh and M. V. Kovalenko, Nano Lett., 2015, 15, 3692-3696.

3 G. Longo, A. Pertegás, L. Martínez-Sarti, M. Sessolo and H. J. Bolink, J. Mater. Chem. C, 2015, 3, 11286-11289.

4 S. Bai, Z. Yuan and F. Gao, J. Mater. Chem. C, 2016, 4, 3898-3904.

5 X. Zhang, H. Lin, H. Huang, C. J. Reckmeier, Y. Zhang, W. C. Choy and A. L. Rogach, Nano Lett., 2016, 16, 1415-1420.

6 S. Yakunin, L. Protesescu, F. Krieg, M. I. Bodnarchuk, G. Nedelcu, M. Humer, G. De Luca, M. Fiebig, W. Heiss and M. V. Kovalenko, Nat. Commun., 2015, 6, 8056.

7 Q. A. Akkerman, V. D’Innocenzo, S. Accornero, A. Scarpellini, A. Petrozza, M. Prato and L. Manna, J. Am. Chem. Soc., 2015, 137, 10276-10281.

8 G. Nedelcu, L. Protesescu, S. Yakunin, M. I. Bodnarchuk, M. J. Grotevent and M. V. Kovalenko, Nano Lett., 2015, 15, 5635-5640.

9 S. Seth, N. Mondal, S. Patra and A. Samanta, J. Phys. Chem. Lett., 2016, 7, 266-271.

10 C. de Weerd, L. Gomez, H. Zhang, W. J. Buma, G. Nedelcu, M. V. Kovalenko and T. Gregorkiewicz, J. Phys. Chem. C, 2016, 120, 13310-13315.

11 Y. S. Park, S. Guo, N. S. Makarov and V. I. Klimov, ACS Nano, 2015, 9, 10386-10393.

12 Q. A. Akkerman, S. G. Motti, A. R. Srimath Kandada, E. Mosconi, V. D’Innocenzo, G. Bertoni, S. Marras, B. A. Kamino, L. Miranda, F. De Angelis, A. Petrozza, M. Prato and L. Manna, J. Am. Chem. Soc., 2016, 138, 1010-1016.

13 J. Shamsi, Z. Dang, P. Bianchini, C. Canale, F. D. Stasio, R. Brescia, M. Prato and L. Manna, J. Am. Chem. Soc., 2016, 138, 7240-7243.

14 H. Huang, B. Chen, Z. Wang, T. F. Hung, A. S. Susha, H. Zhong and A. L. Rogach, Chem. Sci., 2016, 7, 5699-5703. 15 F. Palazon, Q. A. Akkerman, M. Prato and L. Manna, ACS Nano, 2016, 10, 1224-1230.

16 H. Huang, F. Zhao, L. Liu, F. Zhang, X. G. Wu, L. Shi, B. Zou, Q. Pei and H. Zhong, ACS Appl. Mater. Interfaces, 2015, 7, 28128-28133. 
17 F. Palazon, F. Di Stasio, Q. A. Akkerman, R. Krahne, M. Prato and L. Manna, Chem. Mater., 2016, 28, 2902-2906.

18 X. Zhang, B. Xu, J. Zhang, Y. Gao, Y. Zheng, K. Wang and X. W. Sun, Adv. Funct. Mater., 2016, 26, 4595-4600.

19 D. Zhang, S. W. Eaton, Y. Yu, L. Dou and P. Yang, J. Am. Chem. Soc., 2015, 137, 9230-9233.

20 R. J. Sutton, G. E. Eperon, L. Miranda, E. S. Parrott, B. A. Kamino, J. B. Patel, M. T. Horantner, M. B. Johnston, A. A. Haghighirad, D. T. Moore and H. J. Snaith, Adv. Energy Mater., 2016, 6, 1502458.

21 S. R. Raga, M.-C. Jung, M. V. Lee, M. R. Leyden, Y. Kato and Y. Qi, Chem. Mater., 2015, 27, 1597-1603.

22 F. X. Xie, D. Zhang, H. Su, X. Ren, K. S. Wong, M. Gratzel and W. C. Choy, ACS Nano, 2015, 9, 639-646.

23 B. Conings, J. Drijkoningen, N. Gauquelin, A. Babayigit, J. D’Haen, L. D'Olieslaeger, A. Ethirajan, J. Verbeeck, J. Manca, E. Mosconi, F. D. Angelis and H.-G. Boyen, Adv. Energy Mater., 2015, 5, 1500477.
24 R. F. Service, Science, 2016, 351, 113-114.

25 P. Ramasamy, D. H. Lim, B. Kim, S. H. Lee, M. S. Lee and J. S. Lee, Chem. Commun., 2016, 52, 2067-2070.

26 J. De Roo, M. Ibanez, P. Geiregat, G. Nedelcu, W. Walravens, J. Maes, J. C. Martins, I. Van Driessche, M. V. Kovalenko and Z. Hens, ACS Nano, 2016, 10, 2071-2081.

27 B. D. Chernomordik, A. E. Béland, N. D. Trejo, A. A. Gunawan, D. D. Deng, K. A. Mkhoyan and E. S. Aydil, J. Mater. Chem. A, 2014, 2, 10389.

28 L. W. Kun-Hua Wang, L. Li, H.-B. Yao, H.-S. Qian and S.-H. Yu, Angew. Chem., Int. Ed., 2016, 55, 1-6.

29 D. V. Talapin, A. L. Rogach, I. Mekis, S. Haubold, A. Kornowski, M. Haase and H. Weller, Colloids Surf., A, 2002, 202, 145-154.

30 M. Drndić, M. V. Jarosz, N. Y. Morgan, M. A. Kastner and M. G. Bawendi, J. Appl. Phys., 2002, 92, 7498-7503.

31 G. Konstantatos, L. Levina, A. Fischer and E. H. Sargent, Nano Lett., 2008, 8, 1446-1450. 